\title{
Theoretical Study on the Photoelectric Properties of a Class of Copolymers Based on Benzodithiophene for Solar Cells
}

\author{
Xiao-Hua Xie $\mathbb{D}^{1},{ }^{1}$ Xin-Wei Zhao, ${ }^{2}$ and Ming $\mathrm{Li} \mathbb{C}^{3}$ \\ ${ }^{1}$ School of Chemistry and Chemical Engineering, Yangtze Normal University, Chongqing 408100, China \\ ${ }^{2}$ Department of Physics, Tokyo University of Science, 1-3 Kagurazaka, Shinjuku-ku, Tokyo 162-8601, Japan \\ ${ }^{3}$ School of Chemistry and Chemical Engineering, Southwest University, Chongqing 400715, China
}

Correspondence should be addressed to Ming Li; liming@swu.edu.cn

Received 20 January 2018; Accepted 29 March 2018; Published 30 May 2018

Academic Editor: Xiufu Hua

Copyright (c) 2018 Xiao-Hua Xie et al. This is an open access article distributed under the Creative Commons Attribution License, which permits unrestricted use, distribution, and reproduction in any medium, provided the original work is properly cited.

The structural, electronic, and optical properties of PBDTTBT are comprehensively studied by density functional theory to rationalize the experimentally observed properties. Periodic boundary conditions method is employed to simulate the polymer block and calculate effective charge mass from the band structure calculation to describe charge transport properties. Moreover, both time-dependent density functional theory and a set of multidimensional visualization techniques are used to characterize the exciton dissociation ability in the PCBM: PBDTTBT interface. These theoretical methods and calculation techniques not only promote deep understanding of the connection between chemical structures and optical and electronic properties of the donor-acceptor system but also can be used to rationally design a novel donor-acceptor system. Based on the same calculated methods as PBDTTBT, four copolymers PBDTTTP, PBDTTTO, PBDTTTPD, and PBDTTFPD are designed to study their potentials as donors in polymer BHJ. The results indicate that PBDTTBT's well conjugation benefits its good stability, and its wide and strong absorption spectra in the range of visible light, appropriate FMO levels, well charge transport, and favorable exciton dissociation lead to its photovoltaic performance. Furthermore, through comparing the four designed polymers with PBDTTBT, we conclude that the four designed polymers have stronger exciton dissociation ability and larger open-circuit voltage and external quantum efficiencies. Consequently, the four designed copolymers are promising candidates for polymer BHJ solar cells.

\section{Introduction}

Solar cells are one critical technology for solving world energy needs. Traditional solar cells made from high-purity silicon have been commercialized, but their applications are finite because of high cost and weight. Polymer photovoltaic cells with a bulk heterojunction (BHJ) active layer have attracted attention due to their potentially low cost, lightweight, flexible, and easy manufacturing [1-4]. In the BHJ structure, the active layer that is sandwiched between anode and cathode constitutes of electron-donating conjugated polymer and electron acceptor, and its properties are the most determining factors in the whole performance of polymer BHJ [5].

In the past few decades, many progresses have been made in polymer $\mathrm{BHJ}$ which have reached power conversion efficiencies (PCE) of 11\% [6]. The limiting factors of PCE are the stabilities, ability of harvesting the photon flux from the sun, effective exciton separation, and carrier mobilities. In order to improve the device performances, one can develop new device architectures $[7,8]$, synthesize new polymer donors $[9,10]$ and new electron acceptors [11-14], or work on both ends.

In recent years, developing low bandgap polymers has provided an alternative approach for achieving high PCEs resulting from their important roles in photoelectric conversion process: capturing solar photons from the sun, exciton generation and separation, carrier injection, hole transport, and affecting the size of the open-circuit voltage $\left(V_{\mathrm{oc}}\right)$ and short-circuit current $\left(J_{\mathrm{SC}}\right)$, thus affecting PCE $\left(\eta=V_{\mathrm{OC}} \times\right.$ $\left.J_{\mathrm{SC}} \times F F / P_{\text {in }}\right)$. In order to develop low-bandgap conjugated polymers, the most powerful strategy is to incorporate 
electron-rich donor segments and electron-deficient acceptor segments into the polymer backbone. Due to the push-pull interaction, efficient internal charge transfer (ICT) can take place from the donor (D) to the acceptor (A) upon photoexcitation, thus leading to a new absorption band at longer wavelengths [15] and producing appropriate molecular energy levels for high $V_{\mathrm{oc}}$, good charge transport, and high $J_{\mathrm{SC}}$, thus improving PCE [16].

In recent years, some benzo[1,2-b:4,5-b']dithiophenecontaining polymers have been applied to polymer $\mathrm{BHJ}$ and field-effect transistor (FET) [17-22] on account of its electron-rich structures. The relatively large and planar molecular structures of thiophene (TH) derivatives not only keep a high mobility but also help to promote cofacial $\pi-\pi$ stacking. Given the symmetrical planar structure and high mobility of benzo[1,2-b:4,5- $\left.b^{\prime}\right]$ dithiophene (BDT), Huo and his coworkers synthesized a polymer based on BDT, that is, PBDTTBT [23]. PBDTTBT was well soluble in common organic solvents due to its strong octyl chains and presented an excellent thermal stability with a decomposition temperature of $337^{\circ} \mathrm{C}$ without the protection of an inert atmosphere. The group fabricated a polymer solar cell device based on PBDTTBT donor and $\mathrm{PC}_{70} \mathrm{BM}$ (a fullerene derivative) acceptor in 2010. The structure of the device is ITO/PEDOT-PSS/ polymer: $\mathrm{PC}_{70} \mathrm{BM}(1: 2, w / w) / \mathrm{Ca} / \mathrm{Al}$, and its $V_{\mathrm{oc}}, J_{\mathrm{SC}}$, fill factor $(\mathrm{FF})$, and PCE are $0.92 \mathrm{~V}, 10.70 \mathrm{~mA} \cdot \mathrm{cm}^{-2}, 57.5 \%$, and $5.66 \%$, respectively [23].

In order to rationalize the experimentally observed properties of known materials and predict those of unknown ones, theoretical investigations on the structure features, charge transport properties, and exciton dissociation abilities of PBDTTBT are indispensable. In the modeling process, we replace the octyls with butyls to reduce the computation cost. Analogously, we replace benzo[c] $[1,2,5]$ thiadiazole with four pyridazine derivatives and designed four polybenzo[1,2$\left.b: 4,5-b^{\prime}\right]$ dithiophene derivatives, PBDTTTP, PBDTTTO, PBDTTTPD, and PBDTTFPD and study their potentials as donors in polymer $\mathrm{BHJ}$ based on $\mathrm{PC}_{70} \mathrm{BM}$. Several parameters of determining the performance of solar cells, electronic and structural properties, open-circuit voltage $\left(V_{\mathrm{OC}}\right)$, charge transfer properties, exciton dissociation abilities, and theoretical PCE have been investigated.

This paper's distribution is as follows. In Section 2, we describe the computational methods to obtain electronic and structural properties. In Section 3, we detailedly describe the influences on the performance of solar cells by comparing PBDTTBT with designed copolymers, and the conclusion is in Section 4.

\section{Calculation Methods}

Density functional theory (DFT) has been broadly used to investigate the properties of organic compounds because its high accuracy is reasonable with the ab initio method and less computational time cost, and B3LYP, a hybrid functional, is widely used in calculating organic systems [24-27]. In this paper, DFT and time-dependent DFT (TD-DFT) [28] are employed to gain the qualitative properties of all the compounds at b3lyp/6-31g* level $[29,30]$. All the optimized structures are the global minima on the potential energy surface. Periodic boundary conditions (PBC) [31] method is used to optimize block polymers at B3LYP/6-31G(d) and B3PW91/6-31G(d) levels [32]. All the calculations are implemented by Gaussian 09 package [33]. As to the copolymers, density topological analyses are examined by atom in molecule (AIM) analyses [34]. Moreover, the nucleusindependent chemical shift (NICS) $[35,36]$ is also calculated for copolymers at the B3LYP/6-31G* level, and NICS defined as the negative of the magnetic shielding at a ring critical point (RCP) is obtained from the AIM analyses. Furthermore, the bonding characteristics were also investigated by natural bond orbital (NBO) theory [37-40] and AOMix program packages [41].

\section{Results and Discussion}

3.1. Geometry Optimization. The drafts of the studied copolymers are depicted in Figure 1, and the optimized structures of parent molecules of copolymers by B3LYP/6-31G(d) along with the abbreviations of each segments are shown in Figure 2. The selected bond lengths and bond angles of these copolymers are also given in Figure 1; herein, the C1-C2 (in PBDTTBT) or C2-C3 (in other copolymers) bond is defined as the central bond that connects the donor and acceptor. All the studied copolymers have the same central bonds (1.45 $\AA$ ), and their dihedral angles are smaller than $37^{\circ}$, which suggest that all the polymers are rigid backbones. The nitrogen- (N-) hydrogen $(\mathrm{H})$ or sulfur $(\mathrm{S})$ /oxygen- $(\mathrm{O}-)$ nitrogen $(\mathrm{N})$ interactions forming stable six or five-member rings reduce the dihedral angles and keep the molecular coplanarity, thus benefitting to the rigidity of the copolymers. Besides, the densities of bond critical points (BCPs) and RCPs as well as the distances between the two interactional atoms are also presented in Figure 1. It is worth noting that the angles of S1-C4-C5-C6 and C7-C8-C9-S2 in the four designed copolymers are smaller than $\mathrm{C} 4-\mathrm{C} 5-\mathrm{C} 6-\mathrm{C} 7$ and $\mathrm{C} 8-\mathrm{C} 9-$ C10-C11 angles in PBDTTBT, which leads to better coplanarity than PBDTTBT.

In order to acquire the charge population analysis on central bonds in studied polymers, we calculate the characters of bond critical points (BCPs) in central bonds and list the data in Table 1S in Supplementary Materials. The data indicate that the two atoms in central bonds are relatively accumulated due to sharing interactions.

Furthermore, the HOMO and LUMO diagrams of monomers in Figure 1S not only can qualitatively illustrate the electronic cloud distribution but also reflect the electron-donating and electron-accepting segments. We can clearly see from Figure $1 \mathrm{~S}$ that the electronic cloud distribution of HOMO of all the monomers localizes near the polymeric axis and mainly localizes on BDT segments in the four designed copolymers, while that of LUMO localizes near the TBT, TTP, TTO, TTPD, and TFPD segments (the segments in rectangle in Figure 2). The molecular orbital diagrams illustrate that the BDT segments contributing to HOMOs in the five copolymers are as electron-donating segments, and the left segments (TBT, 


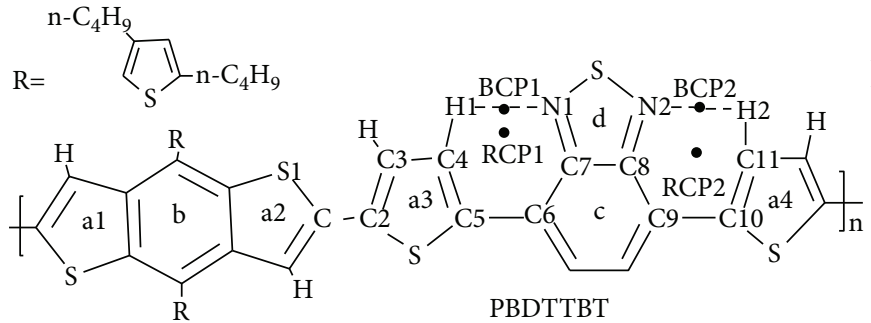

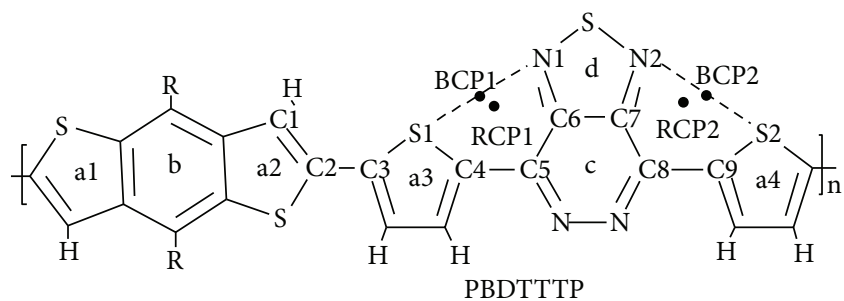

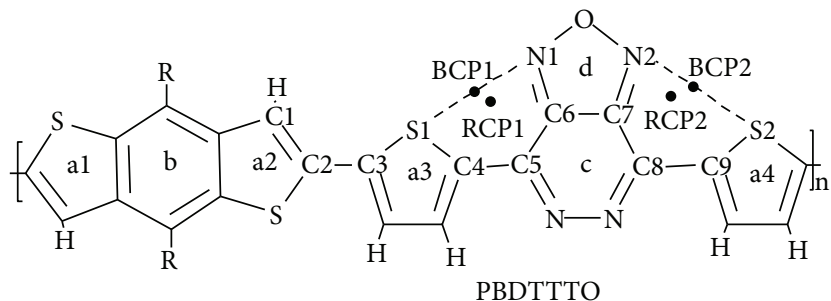

C1-C2: 1.45

N1-H1: 2.56

$\mathrm{N} 2-\mathrm{H} 2: 2.29$

1-C1-C2-C3: $28.69^{\circ}$

C4-C5-C6-C7: $36.79^{\circ}$

C8-C9-C10-C11:18.00

BCP1: 0.011

BCP2: 0.016

RCP1: 0.010

C2-C3: 1.45

N1-S1: 3.17

N2-S2: 3.17

C1-C2-C3-S1: $32.48^{\circ}$

S1-C4-C5-C6: 5.35

C7-C8-C9-S2: $1.81^{\circ}$

BCP1: 0.009

BCP2: 0.009

RCP1: 0.008

RCP2: 0.008

C2-C3: 1.45

N1-S1: 3.29

N2-S2: 3.29

C1-C2-C3-S1: $31.36^{\circ}$

S1-C4-C5-C6: $7.76^{\circ}$

C7-C8-C9-S2: $3.42^{\circ}$

BCP1: 0.009

BCP2: 0.009

RCP1: 0.008

RCP2: 0.008

C2-C3: 1.45

O1-S1: 2.74

O2-S2: 2.74

C1-C2-C3-S1: $19.92^{\circ}$

S1-C4-C5-C6: $2.78^{\circ}$

C7-C8-C9-S2: $3.93^{\circ}$

BCP1: 0.020

BCP2: 0.020

RCP1: 0.008

RCP2: 0.008

C2-C3: 1.45

O1-S1: 2.94

O2-S2: 2.95

C1-C2-C3-S1: $25.50^{\circ}$

$\mathrm{S} 1-\mathrm{C} 4-\mathrm{C} 5-\mathrm{C} 6: 3.82^{\circ}$

C7-C8-C9-S2: $4.36^{\circ}$

BCP1: 0.020

BCP2: 0.020

RCP1: 0.008

RCP2: 0.008

Figure 1: Structural parameters of copolymers and locations for calculated NICS.

TTP, TTO, TTPD, and TFPD) mainly contributing to LUMOs are as electron-accepting segments. Moreover, in order to quantitatively view the components of the HOMOs and LUMOs, the density of state (DOS) and partial DOS (PDOS) of monomers are calculated and given in Figure 3. As shown in Figure 3, TBT segment contributes to both HOMO and LUMO; nevertheless, BDT mainly contributes to HOMO to some degree. The DOS diagrams for other polymers show that the HOMOs are mainly contributed by BDT segments, and the LUMOs are almost entirely from TBT, TTP, TTO, TTPD, and TFPD segments.

In a word, BDT as a donor mainly contributes to HOMO and the left segments mainly contribute to LUMO, and the push-pull interactions in D-A in the four copolymers are formed.

3.2. Conjugational Properties. Generally speaking, favorable structural stability of molecule originates from its favorable conjugated properties to some degree. In order to understand the structural stability of the studied polymers, we investigate their conjugation properties.

NICS is comprehensively used to express the aromaticity of molecules because it can clearly and simply monitor the condition of ring current. Aromatic systems have pretty negative NICS values, antiaromatic systems have strongly positive NICS values, and nonaromatic cyclic systems should 


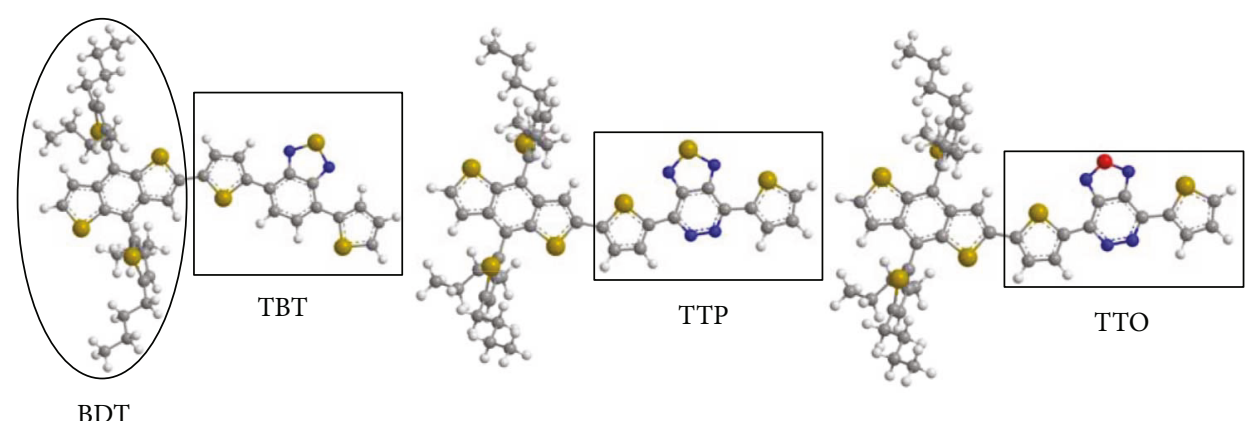

BDTTBT

BDTTTP

BDTTTO

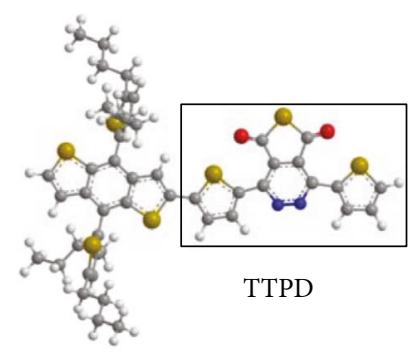

BDTTTPD

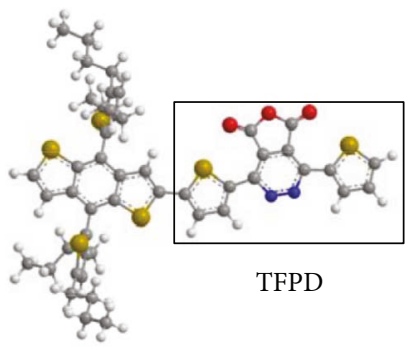

BDTTFPD

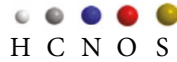

FIGURE 2: The stereograph of optimized parent molecules of copolymers by DFT//B3LYP/6-31G(d).

have NICS values close to zero [35, 42-44]. The NICS values for the repeated units of polymers are calculated and listed in Table $2 \mathrm{~S}$, and the positions of all the rings in the molecules are shown in Figure 1.

In Table 1S, the ring NICS values along the polymeric axis $(a, b$, and $c$ ) are more positive than the individual $\mathrm{TH}$ (NICS, -13.9 at B3LYP/6-31G(d) level) and benzene (NICS, -9.7 at B3LYP/6-31G(d) level), which originates from the electrons delocalizing to the whole molecule, thus, forming conjugated systems. As to the same rings, the NICS values of center rings are more negative than the terminal rings, such as the NICS values in a 2 and a3 rings are more negative than those in a1 and a4, which illustrate that the ring currents mainly accumulate in the molecular center. In addition, the electrons of the molecules delocalize from donors toward acceptors along the polymeric axis because there are push-pull interactions in the systems; therefore, a2, a3, a4, and c rings are full of ring currents but a1 and b rings lack ring currents. Further, inside rings, that is, $\mathrm{d}$ rings, where the NICS values are most negative in respective systems, since electron-rich sulfur and nitrogen atoms in the three systems provide large ring currents. Nevertheless, the carbon atoms in PBDTTTPD and PBDTTFPD are relatively electron-deficient in comparison of nitrogen atoms, which results in small ring currents, thus leading to more positive NICS values in d rings.

Through analyzing the ring currents in RCPs, we find PBDTTTP and PBDTTTO have the same conjugation as PBDTTBT and are relatively stable copolymers.

3.3. Absorption Spectra. As shown in Figure 4(a), the experimental absorption spectrum of PBDTTBT has three absorption bands from 300 to $700 \mathrm{~nm}$ both in chloroform and as a solid film, and the main absorption peak of PBDTTBT is located at approximately $581 \mathrm{~nm}$ in solution [23]. The simulated absorption spectrum of PBDTTBT monomer at the TD-DFT/B3LYP 6-31G(d) level is depicted in Figure 4(a) for comparison, and the effect of the solvent (chloroform) within polarizable continuum model (PCM) [44] is taken into account during the calculation. The main absorption peak of calculated PBDTTBT monomer is located at $607 \mathrm{~nm}$. The agreements between measured and simulated spectra are well in overall spectral shape, though their main absorption peaks are different. Furthermore, the calculated electronic transitions, absorption wavelengths $(\lambda)$, oscillator strengths $(f)$, and main configuration of PBDTTBT monomer were shown in Table 1 . Herein, the $S_{1} \leftarrow S_{0}$ electronic transition mainly comes from HOMO to LUMO (absorption process). In the process of $\mathrm{S}_{1} \leftarrow \mathrm{S}_{0}$ electronic transition, charges transfer from the conjugation section of homocyclic rings along the polymeric axis to TBT section as shown in the HOMO and LUMO diagrams of PBDTTBT in Figure 1S. In comparison of the charge difference densities of the main electronic excited states of PBDTTBT in Figure 2S, we find that the charge transfer takes place from BDT to TBT, whereas the alkyl groups hardly participate in charge transfer.

At the same calculation level, the simulated absorption spectra of the four designed copolymers are given in Figure 4(b). As shown in Figure 4(b), the absorption spectra of monomers for BDTTTP and BDTTTO have two absorption bands in the range of $300-800 \mathrm{~nm}$, especially the absorption bands ( $Q$ band) near to $700 \mathrm{~nm}$, thus benefitting to harvesting influx photons from the sun, and the monomers 

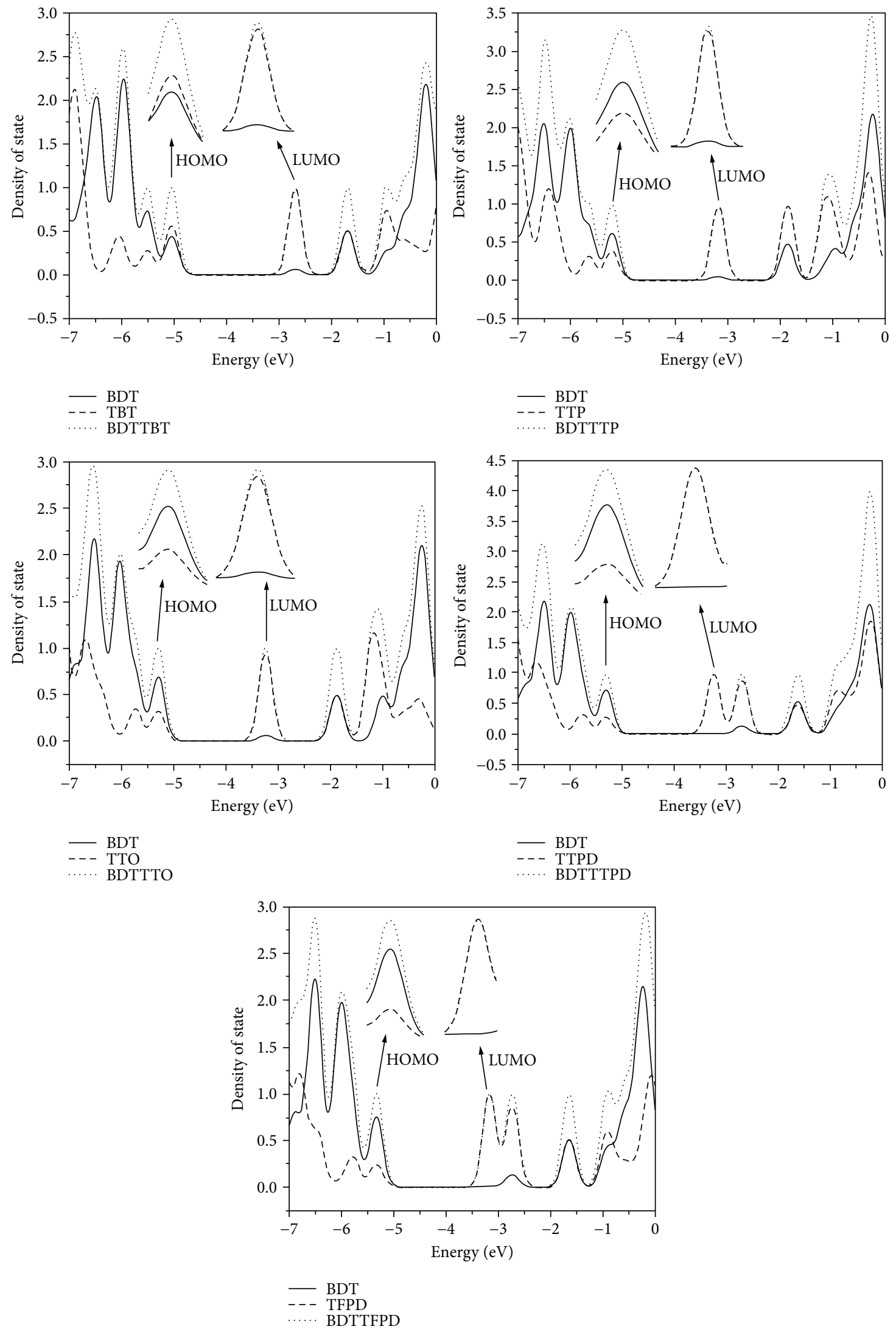

FIgURE 3: DOS and PDOS of monomers. 


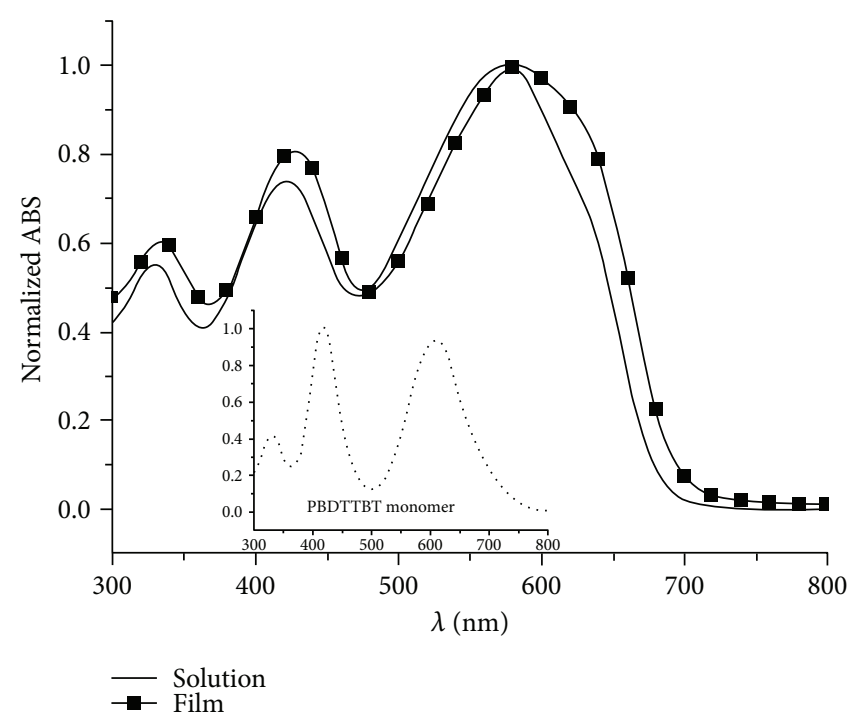

(a)

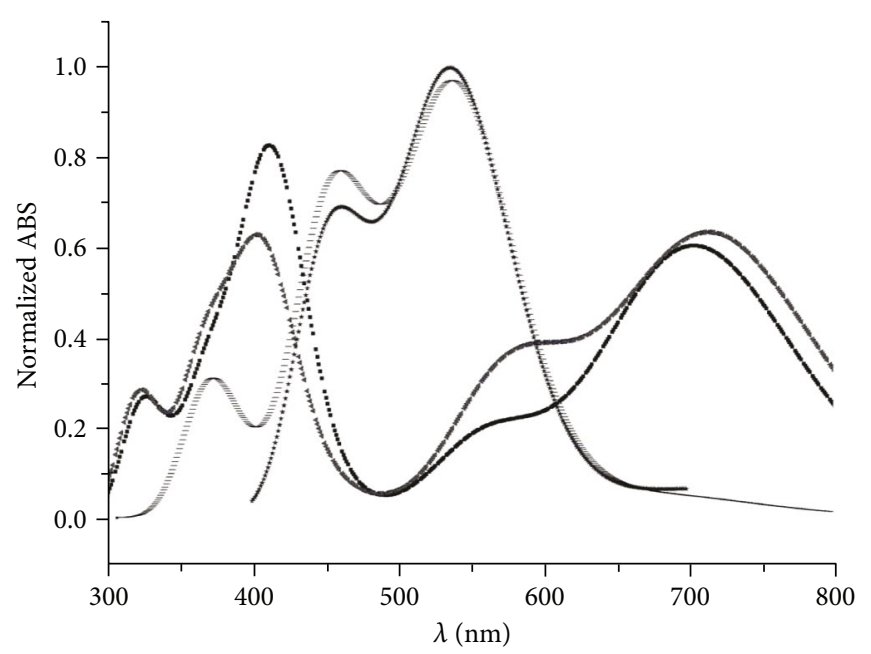

- BDTTTP

BDTTTO
* BDTTTPD

(b)

FIgURE 4: (a) Experimental absorption spectra of PBDTTBT as a film and in chloroform solution [23] and simulated absorption spectra of PBDTTBT monomer (the smaller one) in chloroform solution. (b) Absorption spectra of monomers of the four designed copolymers in chloroform solution.

of BDTTTPD and BDTTFPD have wide absorption bands in the range of $400-600 \mathrm{~nm}$. The above results suggest that the parent molecules of designed copolymers have broad absorption in the visible region.

Moreover, the detailed maximum absorption wavelengths $(\lambda)$, oscillator strengths $(f)$, the lowest excitation energy $\left(E^{\mathrm{ex}}\right)$, and the main configuration of monomers are given in Table 1. In Table 1, the maximum absorption peaks of BDTTTP and BDTTTO belonging to Q bands are redshifted compared with BDTTBT and the $E^{\mathrm{ex}}$ values decrease, thus benefitting electronic transition, whereas, these of BDTTTPD and BDTTFPD are blue-shifted. Generally speaking, the larger oscillator strengths $(f)$, the larger electronic transition probability. As shown in Table 1, the oscillator strengths $(f)$ of all the studied monomers are larger than 0.61 , and that of BDTTTPD is the largest one (0.96) of all the $f$. Combining Figure 4 and Table 1, we find that the main configurations with the maximum wavelength of the parent molecules belong to single electron transitions and originate from HOMO to LUMO mainly assigned to $\pi \rightarrow \pi^{*}$ transition; as shown in Figure 1S, the electrons transfer from donor (BDT) to acceptors (TBT, TTP, TTO, TTPD, and TFPD).

In comparison to the experimental and theoretical absorption spectra of BDTTBT, we find that the PBDTTBT has wide and strong absorption spectra. Moreover, the designed copolymers have wide and strong absorption, and they have smaller transition energy in the visible region. Therefore, these designed copolymers also have the favorable ability of harvesting the flux photons from the sun.

3.4. Frontier Molecular Orbitals. The properties of frontier molecular orbitals (FMOs) of polymers seriously affect stable and photovoltaic properties. In order to harvest the maximum of the photon flux from the sun and obtain high short-circuit current $\left(J_{\mathrm{SC}}\right)$, the bandgap $(\mathrm{Eg})$ of the polymers should lie between 1.3 and $1.9 \mathrm{eV}$ [45]. Further, its HOMO energy level should be between -5.2 and $-5.8 \mathrm{eV}$ if the donor can keep stable toward oxidation from air, meanwhile, its LUMO level should be between -3.7 and $-4.0 \mathrm{eV}$ as shown in Figure 5. Rather, the open-circuit voltage $\left(V_{O C}\right)$ of PSC is eventually confined by the difference between the HOMO of the donor and the LUMO of the acceptor $[12,46]$. It is useful to research the molecular FMOs because the relative levels of the occupied and virtual orbitals can provide reasonable qualitative indications for processes of exciton generation and dissociation.

In the present work, we calculate the LUMO and HOMO energy levels of the five copolymers by exploring several DFT methods. Since only the values at B3LYP/6-31G(d) and B3PW91/6-31G(d) levels get close to the experimental values of PBDTTBT (the detailed calculated results are given in Table 3S in Supplementary materials); therefore, we only list the LUMO and HOMO energy levels based on the above methods in Figure 5.

In Figure 5, we can see that the value at the B3LYP/6$31 \mathrm{G}(\mathrm{d})$ level $(-5.01 \mathrm{eV})$ is higher than the one at B3PW91/ 6-31G(d) level $(-5.11 \mathrm{eV})$, and the two calculated values are higher than the experimental one $(-5.31 \mathrm{eV})$. The difference between experimental and calculated values is about $0.2-0.3 \mathrm{eV}$, and the different environments between experiment and calculation should be responsible for the difference. As to the designed copolymers, the HOMO levels from B3LYP/6-31G(d) level are higher than the corresponding ones from B3PW91/6-31G(d) level as the PBDTTBT; moreover, all the HOMO levels of designed copolymers are lower than the one in PBDTTBT at the same calculated level; therefore, we predict that the experimental HOMO levels of the designed copolymers are lower than the experimental one of PBDTTBT $(-5.31 \mathrm{eV})$, and the designed copolymers have stronger antioxidation properties than PBDTTBT. 
TABLE 1: Maximum absorption wavelengths $(\lambda)$, oscillator strengths $(f)$, the lowest excitation energies, and main configuration of monomers.

\begin{tabular}{lcccc}
\hline Monomer & $\lambda(\mathrm{nm})$ & $f$ & $E^{\mathrm{ex}}(\mathrm{eV})$ & Main configuration \\
\hline BDTTBT & 607 & 0.81 & 2.04 & $\mathrm{HOMO} \rightarrow \mathrm{LUMO}(99 \%)$ \\
BDTTTP & 706 & 0.61 & 1.76 & $\mathrm{HOMO} \rightarrow \mathrm{LUMO}(99 \%)$ \\
BDTTTO & 718 & 0.63 & 1.73 & $\mathrm{HOMO} \rightarrow \mathrm{LUMO}(100 \%)$ \\
BDTTTPD & 538 & 0.96 & 2.30 & $\mathrm{HOMO} \rightarrow \mathrm{L}+1(97 \%)$ \\
BDTTFPD & 540 & 0.85 & 2.30 & $\mathrm{HOMO} \rightarrow \mathrm{L}+1(83 \%)$ \\
\hline
\end{tabular}

In the formula of power conversion efficiency: $\eta=V_{\mathrm{OC}} \times$ $J_{\mathrm{SC}} \times F F / P_{\text {in }}$, the three parameters, $V_{\mathrm{OC}}, J S C$, and $F F$, determine the solar cell performance directly. The parameter, open-circuit voltage $\left(V_{\mathrm{OC}}\right)$ formed in the process of carrier transport, is popularly used to estimate the maximum PCE [9]. As shown in Figure 5, the experimental $V_{\mathrm{OC}}$ (exp) is gained when the $J_{\mathrm{SC}}$ is equal to zero in $\mathrm{J}-\mathrm{V}$ curves. There are two models to describe theoretical $V_{\mathrm{OC}}$ : one is the metalinsulator-metal (MIM) model [47], the other model is the $\mathrm{HOMO}^{\mathrm{D}}$-LUMO ${ }^{\mathrm{A}}$ offset model $[9,48]$. Moreover, Lo et al. suggest that $V_{\text {oc }}$ is described by a combined MIM model with $\mathrm{HOMO}^{\mathrm{D}}$-LUMO ${ }^{\mathrm{A}}$ offset model; in that model, they find that when the work function deviation $\left(\Delta \phi_{\text {electrodes }}\right)$ of ITO from $\mathrm{Al}$ electrode is in the range- 3 and $0 \mathrm{eV}, V_{\mathrm{oc}}$ enlarges linearly with $\Delta \phi_{\text {electrodes }}$ as prescribed by the MIM model. Outside this range $V_{\text {oc }}$ depends on $\mathrm{HOMO}^{\mathrm{D}}$ LUMO $^{\mathrm{A}}$ offset model [49]. In the work, we assume that $\Delta \phi_{\text {electrodes }}$ is outside the range -3 and $0 \mathrm{eV}$; thus, we employed the $\mathrm{HOMO}^{\mathrm{D}}$-LUMO ${ }^{\mathrm{A}}$ offset model, and the $V_{\text {oc }}$ of a conjugated polymer- $\mathrm{PC}_{70} \mathrm{BM}$ solar cell can be estimated by [9]

$$
V_{\mathrm{oc}}=\frac{1}{e}\left(\left|E^{\text {Donor }} \mathrm{HOMO}\right|-\left|E^{\mathrm{PCBM}} \mathrm{LUMO}\right|\right)-0.3 \mathrm{~V} \text {, }
$$

where $e$ is the elementary charges, $E^{\mathrm{PCBM}}$ LUMO is equal to $-4.3 \mathrm{eV}\left(\mathrm{PC}_{70} \mathrm{BM}\right)$, and the $0.3 \mathrm{~V}$ is an empirical factor to offset the exciton binding energy [50]. In comparison with the experimental and simulated $V_{\mathrm{OC}}$ values of PBDTTBT, the simulated $V_{\mathrm{OC}}$ is lower than the measured one by 0.51 (at the B3LYP/6-31G* level) and $0.41 \mathrm{eV}$ (at the B3PW91/ $6-31 G^{*}$ level), which primarily originates from the HOMO level difference between simulation and experiment. For the sake of comparing the $V_{\mathrm{OC}}$ size of designed polymers with PBDTTBT, we also simulate the $V_{\mathrm{OC}}$ values of designed polymers by $\mathrm{HOMO}^{\mathrm{D}}$-LUMO ${ }^{\mathrm{A}}$ offset model. As shown in Figure 5, the $V_{\text {oc }}$ of the designed copolymers are larger than the one of PBDTTBT based on either B3LYP or B3PW91 functional resulting from the deeper HOMO levels of designed copolymers. Therefore, the four designed copolymers are promising to improve the $V_{\text {oc }}$ relative to PBDTTBT in the experiment.

3.5. Charge Transfer Properties. On the basis of band-like theory, the bandwidth (BW) and electron effective mass $\left(m^{*}\right)$ are beneficial parameters for predicting the hole and electron-transporting ability of polymers [51-53]. The effective mass of carrier at the band edge representing mobility was gained as the square of $\hbar$ multiplied by the reciprocal of the curvature from $E(k)$ with $k$, and the formulation is defined as

$$
\frac{1}{m^{*}}=\frac{1}{\hbar}\left(\frac{\partial^{2} E(k)}{\partial k^{2}}\right) .
$$

The kinetic model of mobility $(\mu)$ is given by the following formula:

$$
\mu=\frac{e T}{m^{*}} .
$$

The BW and $m^{*}$ data are given in Table 2. According to the band theory, the wider the BW, the smaller the effective mass, and the larger the kinetic model of mobility [54]. The data in Table 2 investigate that PBDTTBT has wide valence band $(0.25 \mathrm{eV})$ and small $m^{*}\left(-4.45 \times 10^{4}\right)$. By using the same methods as PBDTTBT, we calculate the bandwidths and effective masses for designed polymers and list the data in Table 2 .

The data in Table 2 investigate that all the designed polymers have wider valence bands (PBDTTTP: $0.38 \mathrm{eV}$, PBDTTTO: $0.34 \mathrm{eV}$, PBDTTTPD: $0.33 \mathrm{eV}$, and PBDTTFPD: $0.30 \mathrm{eV}$ ) and smaller $m^{*}$ (PBDTTTP: $-2.91 \times 10^{4}$, PBDTTTO: $-2.91 \times 10^{4}$, PBDTTTPD: $-2.91 \times 10^{4}$, and PBDTTFPD: $-3.29 \times 10^{4}$ ) in comparison of PBDTTBT. Moreover, PBDTTTP and PBDTTTO also have wider conduction bands and smaller $m^{*}$ than PBDTTBT; however, PBDTTTPD and PBDTTFPD have narrower conduction bandwidths and valence bandwidths through comparing PBDTTBT. The above investigations indicate that all the designed polymers have better hole transport properties than PBDTTBT; further, PBDTTTP and PBDTTTO have better electron transport properties than PBDTTBT through conduction band properties.

3.6. Exciton Dissociation. After exciton formation, excitons transport to the donor-acceptor interface. In the interface, exciton dissociation competes with possible recombination. The rates of exciton dissociation and charge recombination are evaluated using the Marcus theory [55]:

$$
K=\sqrt{\frac{4 \pi^{3}}{h^{2} \lambda k_{\mathrm{B}} T}}\left|V_{\mathrm{DA}}\right|^{2} \exp \left(-\frac{(\Delta G+\lambda)^{2}}{4 \lambda k_{\mathrm{B}} T}\right),
$$

where $\lambda$ is the reorganization energy, $V_{\mathrm{DA}}$ is the electronic coupling between donor and acceptor, $\Delta G$ is the free energy change for the electron transfer reaction, $k_{\mathrm{B}}$ is the Boltzmann constant, $h$ is the Planck's constant, and $T$ is the temperature (we set $T=298 \mathrm{~K}$ in our calculations). In the exciton dissociation and charge recombination, $\Delta G=\Delta G_{\mathrm{CT}}$ and $\Delta G=\Delta G_{\mathrm{CR}}$, respectively. The electronic coupling can be calculated through employing the generalized Mulliken-Hush (GMH) model [56]. In the work, we calculate the ratio of exciton dissociation rate $\left(K_{\mathrm{CT}}\right)$ and charge recombination rate $\left(K_{\mathrm{CR}}\right)$; therefore, the parameters $\lambda, \Delta G_{\mathrm{CT}}$, and $\Delta G_{\mathrm{CR}}$ are mainly studied.

The reorganization energy $\lambda$ is composed of inner and outer reorganization energy. The inner originates from the 


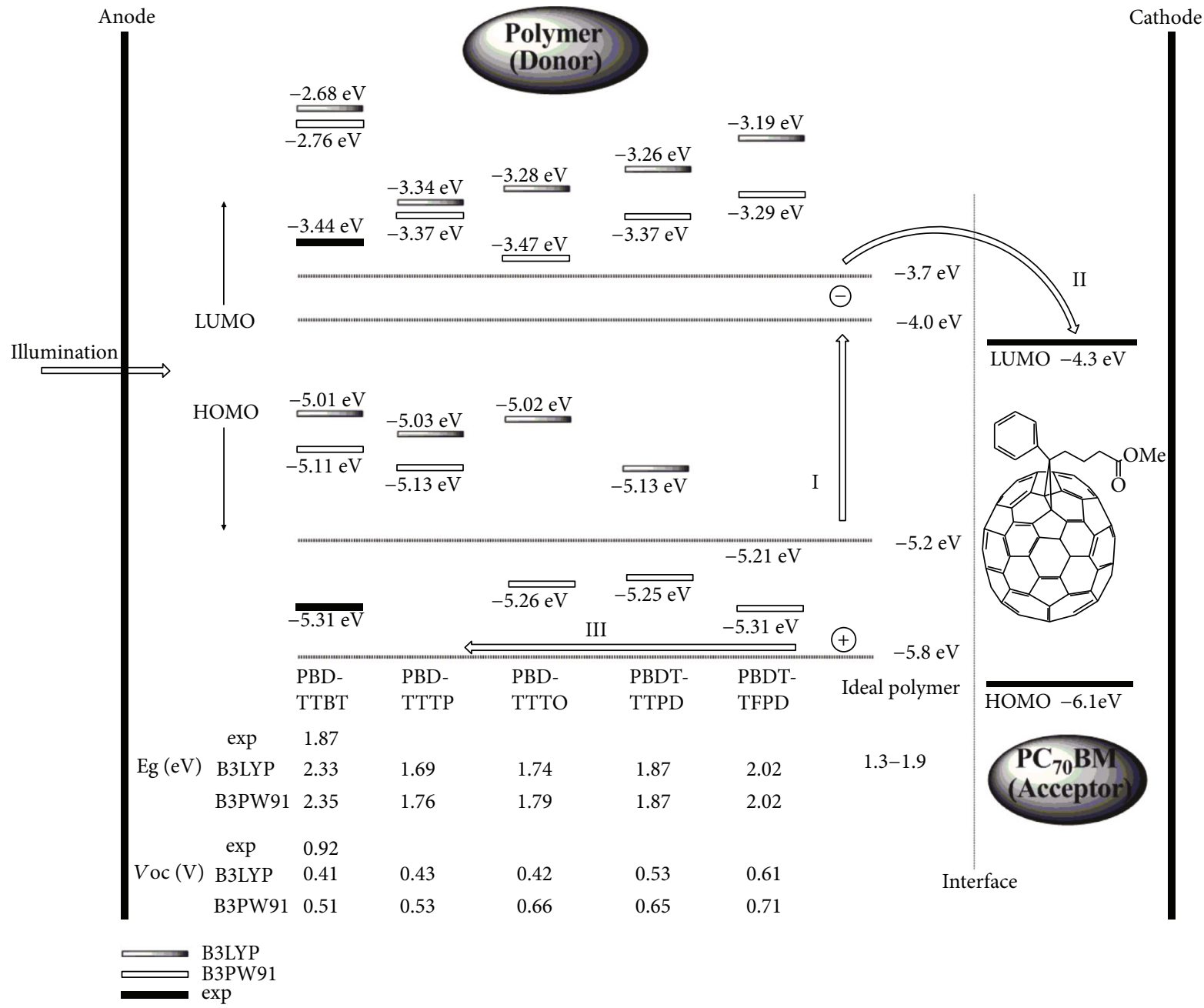

FIGURE 5: Photoelectric conversion process diagram and energy levels of polymers. (exp represents the values of the experimental measures).

TABle 2: Bandwidth (eV) and effective mass $\left(m^{*}\right)$.

\begin{tabular}{lcccc}
\hline \multirow{2}{*}{ Polymer } & \multicolumn{2}{c}{ Conduction band } & \multicolumn{2}{c}{ Valance band } \\
& BW & $m^{*}\left(\times 10^{4}\right)$ & BW & $m^{*}\left(\times 10^{4}\right)$ \\
\hline PBDTTBT & 0.08 & 9.45 & 0.25 & -4.45 \\
PBDTTTP & 0.13 & 5.82 & 0.38 & -2.91 \\
PBDTTTO & 0.16 & 5.04 & 0.34 & -2.91 \\
PBDTTTPD & 0.03 & 12.6 & 0.33 & -2.91 \\
PBDTTFPD & 0.03 & 12.6 & 0.30 & -3.29 \\
\hline
\end{tabular}

change in equilibrium geometry of the donor (D) and acceptor (A) sites consecutive to the gain or loss of electronic charge upon electron transfer. The outer arises from electronic and nuclear polarization/relaxation of the surrounding medium. The inner is composed of two terms

$$
\begin{aligned}
\lambda & =\lambda_{1}(\mathrm{~A})+\lambda_{2}(\mathrm{D}), \\
\lambda_{1}(\mathrm{~A}) & =E\left(\mathrm{~A}^{-}\right)-E(\mathrm{~A}), \\
\lambda_{2}(\mathrm{D}) & =E(\mathrm{D})-E\left(\mathrm{D}^{+}\right),
\end{aligned}
$$

Here, $E\left(\mathrm{~A}^{-}\right)$and $E(\mathrm{~A})$ are the energies of the neutral acceptor $\mathrm{A}$ at the anionic geometry and optimal groundstate geometry, respectively, and $E(\mathrm{D})$ and $E\left(\mathrm{D}^{+}\right)$are, accordingly, the energies of the radical cation at the neutral geometry and optimal cation geometry. In the condition of corrole-fullerene dyads in nonpolar solvent, the overall reorganization energy is $0.5 \mathrm{eV}$. Therefore, we assume here a value of $0.5 \mathrm{eV}$ for the overall reorganization energy in our calculations. All the reorganization energies of researched polymers are given in Table 3 . The reorganization energy of PBDTTBT, PBDTTTP, PBDTTTO, and PBDTTFPD is $0.69 \mathrm{eV}$ except for $0.67 \mathrm{eV}$ of PBDTTTPD. The $\Delta G_{\mathrm{CR}}$ can be estimated with

$$
\Delta G_{\mathrm{CR}}=E I_{P}(\mathrm{D})-E_{\mathrm{EA}}(\mathrm{A}),
$$

where $E_{\mathrm{IP}}(\mathrm{D})$ and $E_{\mathrm{EA}}(\mathrm{A})$ are the ionization potential of the donor and electron affinity of the acceptor, respectively. The calculated $\Delta \mathrm{G}_{\mathrm{CR}}$ values are successively $-1.76 \mathrm{eV},-1.92 \mathrm{eV}$, $-2.10 \mathrm{eV},-2.09 \mathrm{eV},-2.15 \mathrm{eV}$ for PBDTTBT, PBDTTTP, PBDTTTO, PBDTTTPD, PBDTTFPD shown in Table 3. 
TABLE 3: Reorganization $(\lambda)$, exciton dissociation energy $\left(\Delta G_{\mathrm{CT}}\right)$, charge recombination energy $\left(\Delta G_{\mathrm{CR}}\right)$, the ratio of exciton dissociation rate and charge recombination rate $\left(K_{\mathrm{CT}}: K_{\mathrm{CR}}\right)$, and external quantum efficiency (EQE).

\begin{tabular}{lccccc}
\hline Polymer & PBDTTBT & PBDTTTP & PBDTTTO & PBDTTTPD & PBDTTFPD \\
\hline$\lambda(\mathrm{eV})$ & 0.69 & 0.69 & 0.69 & 0.67 & 0.69 \\
$\Delta G_{\mathrm{CR}}(\mathrm{eV})$ & -1.76 & -1.92 & -2.10 & -2.09 & -2.15 \\
$\Delta G_{\mathrm{CT}}(\mathrm{eV})$ & -0.31 & -0.14 & -0.29 & -0.21 & -0.15 \\
$K_{\mathrm{CT}}: K_{\mathrm{CR}}$ & $1.62 \times 10^{6}$ & $2.36 \times 10^{7}$ & $2.13 \times 10^{11}$ & $1.90 \times 10^{11}$ & $2.70 \times 10^{11}$ \\
$\mathrm{EQE}$ & $1.3 \%$ & $4.1 \%$ & $3.8 \%$ & $3.7 \%$ & $3.6 \%$ \\
\hline
\end{tabular}

As shown in Table 3 , we also estimated the $\Delta \mathrm{G}_{\mathrm{CT}}$ values in accordance with Rehm-Weller equation [57]

$$
\Delta G_{C T}=-\Delta G_{C R}-\Delta E_{0-0}-E_{b}
$$

where $\Delta E_{0-0}$ is the energy of the lowest excited state of freebase donor and $E_{\mathrm{b}}$ is the exciton binding energy. The calculated $\Delta G_{\mathrm{CT}}$ are depicted in Table 3 , and they are $-0.31 \mathrm{eV}$ (PBDTTBT), $-0.14 \mathrm{eV}$ (PBDTTTP), $-0.29 \mathrm{eV}$ (PBDTTTO), $-0.21 \mathrm{eV}$ (PBDTTTPD), and $-0.15 \mathrm{eV}$ (PBDTTFPD). According to the estimated values of $\lambda, \Delta G_{\mathrm{CR}}$, and $\Delta G_{\mathrm{CT}}$, we calculate the ratio of exciton dissociation rate and charge recombination rate $\left(K_{\mathrm{CT}}: K_{\mathrm{CR}}\right)$, and the data of $K_{\mathrm{CT}}: K_{\mathrm{CR}}$ listed in Table 3 indicate that PBDTTBT has large $K_{\mathrm{CT}}: K_{\mathrm{CR}}$ $\left(1.62 \times 10^{6}\right)$. Moreover, the designed polymers PBDTTTP, PBDTTTO, PBDTTPD, and PBDTTFPD have larger $K_{\mathrm{CT}}: K_{\mathrm{CR}}$ values $\left(2.36 \times 10^{7}, 2.13 \times 10^{11}, 1.90 \times 10^{11}\right.$, and $2.70 \times 10^{11}$, resp.) than PBDTTBT, which suggest that the designed polymers have better exciton abilities than PBDTTBT.

3.7. External Quantum Efficiency. According to the studies of Scharber et al. in 2006 [9], the external quantum efficiency (EQE) of solar cell is related to the bandgap energy and frontier molecular orbital energy levels under the assumptions that any contribution to the short-circuit current from photons absorbed by the fullerene is neglected and the $F F$ is set to $65 \%$. The calculated EQE data of all the studied polymers are listed in Table 3. From Table 3, we can see that the EQE values of all the designed polymers are larger than $3.5 \%$ (especially PBDTTTP: 4.1\%) and larger than that of PBDTTBT (1.3\%).

In a word, the designed polymers, especially PBDTTTP, have better photovoltaic properties than PBDTBT; therefore, the designed polymers are the promising candidates for polymer BHJ.

\section{Conclusions}

In this contribution, density functional theory (DFT) and time-dependent DFT (TD-DFT) calculations have been employed to model PBDTTBT to rationalize the relationship between the experimentally observed properties and the structural features in polymer BHJ. Besides, according to PBDTTBT, we design four benzodithiophene-like copolymers, PBDTTTP, PBDTTTO, PBDTTTPD, and PBDTTFPD. In order to investigate their potentials as donors in polymer BHJ based on PC70BM, several parameters of determining the performance of solar cells, structural properties, absorption spectra, frontier molecular orbitals, charge transfer properties, and external quantum efficiency have been researched. PBDTTBT's well conjugation benefits its good stability, and its well photovoltaic performance mainly results from its strong absorption spectra in the range of visible light, appropriate FMO levels, well charge transport, and favorable exciton dissociation. Comparing the four designed copolymers with PBDTTBT, we conclude that the four designed copolymers have stronger exciton dissociation ability and larger open-circuit voltage and external quantum efficiencies. Consequently, the four designed copolymers are promising candidates for polymer BHJ solar cells.

\section{Data Availability}

The data used to support the findings of this study are available from the corresponding author upon request.

\section{Conflicts of Interest}

The authors declare that they have no conflicts of interest.

\section{Acknowledgments}

This work was supported by the Research Project from Chongqing Committee of Education (KJ15012020), "Chunhui Plan" Cooperating Project from the Ministry of Education (Z2015143), Natural Science Foundation of Yangtze Normal University (Grant no.2103XJQN007), and Chinese Scholarship Council. The authors are grateful to the anonymous referees for their suggestions.

\section{Supplementary Materials}

Table 1S: BCP properties, Wiberg bond index, and electronic configurations for bonding orbitals of central bonds. Table 2S: NICS for polymers at RCPs. Table 3S: HOMO and LUMO energy levels and bandgaps from the experiment and calculation for PBDTTBT. Figure 1S: $\mathrm{HOMO}(\mathrm{H})$ and LUMO (L) diagrams for monomers and dimers. Figure $2 \mathrm{~S}$ : charge difference densities of the main electronic excited states of PBDTTBT. Figure 3S: orbital interaction diagrams (B3LYP/6-31G *) for dimers. (Supplementary Materials)

\section{References}

[1] F. C. Krebs, M. Jorgensen, K. Norrman et al., "A complete process for production of flexible large area polymer solar cells entirely using screen printing-first public demonstration," 
Solar Energy Materials and Solar Cells, vol. 93, no. 4, pp. 422441, 2009.

[2] F. C. Krebs, "Polymer solar cell modules prepared using rollto-roll methods: knife-over-edge coating, slot-die coating and screen printing," Solar Energy Materials and Solar Cells, vol. 93, no. 4, pp. 465-475, 2009.

[3] F. C. Krebs, S. A. Gevorgyan, and J. Alstrup, "A roll-to-roll process to flexible polymer solar cells: model studies, manufacture and operational stability studies," Journal of Materials Chemistry, vol. 19, no. 30, pp. 5442-5451, 2009.

[4] F. C. Krebs, "All solution roll-to-roll processed polymer solar cells free from indium-tin-oxide and vacuum coating steps," Organic Electronics, vol. 10, no. 5, pp. 761-768, 2009.

[5] B. C. Thompson and J. M. J. Fréchet, "Polymer-fullerene composite solar cells," Angewandte Chemie International Edition, vol. 47, no. 1, pp. 58-77, 2008.

[6] C. Liang, Y. Wang, D. Li, X. Ji, F. Zhang, and Z. He, "Modeling and simulation of bulk heterojunction polymer solar cells," Solar Energy Materials and Solar Cells, vol. 127, no. 4, pp. 67-86, 2014.

[7] A. Hadipour, B. de Boer, J. Wildeman et al., "Solutionprocessed organic tandem solar cells," Advanced Functional Materials, vol. 16, no. 14, pp. 1897-1903, 2006.

[8] J. Y. Kim, K. Lee, N. E. Coates et al., "Efficient tandem polymer solar cells fabricated by all-solution processing," Science, vol. 317, no. 5835, pp. 222-225, 2007.

[9] M. C. Scharber, D. Mühlbacher, M. Koppe et al., "Design rules for donors in bulk-heterojunction solar cells-towards $10 \%$ energy-conversion efficiency," Advanced Materials, vol. 18, no. 6, pp. 789-794, 2006.

[10] E. Bundgaard and F. C. Krebs, "Low band gap polymers for organic photovoltaics," Solar Energy Materials and Solar Cells, vol. 91, no. 11, pp. 954-985, 2007.

[11] M. M. Wienk, J. M. Kroon, W. J. H. Verhees et al., "Efficient methano[70]fullerene/MDMO-PPV bulk heterojunction photovoltaic cells," Angewandte Chemie International Edition, vol. 42, no. 29, pp. 3371-3375, 2003.

[12] C. J. Brabec, A. Cravino, D. Meissner et al., "Origin of the open circuit voltage of plastic solar cells," Advanced Functional Materials, vol. 11, no. 5, pp. 374-380, 2001.

[13] S. A. Backer, K. Sivula, D. F. Kavulak, and J. M. J. Frechet, "High efficiency organic photovoltaics incorporating a new family of soluble fullerene derivatives," Chemistry of Materials, vol. 19, no. 12, pp. 2927-2929, 2007.

[14] F. B. Kooistra, J. Knol, F. Kastenberg et al., "Increasing the open circuit voltage of bulk-heterojunction solar cells by raising the LUMO level of the acceptor," Organic Letters, vol. 9, no. 4, pp. 551-554, 2007.

[15] A. J. Campbell, D. D. C. Bradley, and H. Antoniadis, "Dispersive electron transport in an electroluminescent polyfluorene copolymer measured by the current integration time-of-flight method," Applied Physics Letters, vol. 79, no. 14, pp. 21332135, 2001.

[16] Y. J. Cheng, S. H. Yang, and C. S. Hsu, "Synthesis of conjugated polymers for organic solar cell applications," Chemical Reviews, vol. 109, no. 11, pp. 5868-5923, 2009.

[17] J. Hou, H.-Y. Chen, S. Zhang et al., "Synthesis of a low band gap polymer and Its application in highly efficient polymer solar cells," Journal of the American Chemical Society, vol. 131, no. 43, pp. 15586-15587, 2009.
[18] H.-Y. Chen, J. Hou, S. Zhang et al., "Polymer solar cells with enhanced open-circuit voltage and efficiency," Nature Photonics, vol. 3, no. 11, pp. 649-653, 2009.

[19] H. Pan, Y. Li, Y. Wu et al., "Low-temperature, solutionprocessed, high-mobility polymer semiconductors for thinfilm transistors," Journal of the American Chemical Society, vol. 129, no. 14, pp. 4112-4113, 2007.

[20] H. Pan, Y. Wu, Y. Li et al., "Benzodithiophene copolymer-a low-temperature, solution-processed high-performance semiconductor for thin-film transistors," Advanced Functional Materials, vol. 17, no. 17, pp. 3574-3579, 2007.

[21] J. Hou, M.-H. Park, S. Zhang, L.-M. Chen, J.-H. Li, and Y. Yang, "Bandgap and molecular energy level control of conjugated polymer photovoltaic materials based on benzo[1,2$b: 4,5-b^{\prime}$ ]dithiophene," Macromolecules, vol. 41, no. 16, pp. 6012-6018, 2008.

[22] L. Huo, J. Hou, H.-Y. Chen et al., "Bandgap and molecular level control of the low-bandgap polymers based on 3,6Dithiophen-2-yl-2,5-dihydropyrrolo[3,4-c]pyrrole-1,4-dione toward highly efficient polymer solar cells," Macromolecules, vol. 42, no. 17, pp. 6564-6571, 2009.

[23] L. Huo, J. Hou, S. Zhang, H.-Y. Chen, and Y. Yang, "A polybenzo[1,2-b:4,5- $b^{\prime}$ ]dithiophene derivative with deep HOMO level and its application in high-performance polymer solar cells," Angewandte Chemie International Edition, vol. 49, no. 8, pp. 1500-1503, 2010.

[24] I. T. Lima, L. Sousa, R. d. S. Freitas, L. A. Ribeiro Junior, R. T. de Sousa Júnior, and D. A. de Silva Filho, "A DFT study of a set of natural dyes for organic electronics," Journal of Molecular Modeling, vol. 23, no. 12, p. 343, 2017.

[25] T. Mohr, V. Aroulmoji, R. S. Ravindran et al., "DFT and TD-DFT study on geometries, electronic structures and electronic absorption of some metal free dye sensitizers for dye sensitized solar cells," Spectrochimica Acta Part A: Molecular and Biomolecular Spectroscopy, vol. 135, pp. 1066-1073, 2015.

[26] Y. Li, T. Pullerits, M. Zhao, and M. Sun, "Theoretical characterization of the $\mathrm{PC}_{60} \mathrm{BM}$ :PDDTT Model for an organic solar cell," The Journal of Physical Chemistry C, vol. 115, no. 44, pp. 21865-21873, 2011.

[27] X. H. Xie, W. Shen, R. X. He, and M. Li, “A density functional study of furofuran polymers as potential materials for polymer solar cells," Bulletin of the Korean Chemical Society, vol. 34, no. 10, pp. 2995-3004, 2013.

[28] G. Parr and W. Yang, Density-Functional Theory of Atoms and Molecules, University Press, Oxford, NY, USA, 1989.

[29] A. D. Becke, "Density-functional thermochemistry. III. The role of exact exchange," The Journal of Chemical Physics, vol. 98, no. 7, pp. 5648-5652, 1993.

[30] C. Lee, W. Yang, and R. G. Parr, "Development of the ColleSalvetti correlation-energy formula into a functional of the electron density," Physical Review B, vol. 37, no. 2, pp. 785789, 1988.

[31] K. N. Kudin and G. E. Scuseria, "Linear-scaling densityfunctional theory with Gaussian orbitals and periodic boundary conditions: efficient evaluation of energy and forces via the fast multipole method," Physical Review B, vol. 61, no. 24, pp. 16440-16453, 2000.

[32] J. P. Perdew, Electronic Structure of Solids '91, P. Ziesche and H. Eschrig, Eds., Akademie Verlag, Berlin, 1991.

[33] M. J. Frisch, G. W. Trucks, H. B. Schlegel et al., Gaussian 09, Revision A. 02, Gaussian, Inc., Wallingford, CT, USA, 2009. 
[34] R. F. W. Bader, Atoms in Molecules, A Quantum Theory; International Series of Monographs in Chemistry, vol. 22, Oxford University Press, Oxford, UK, 1990.

[35] P. v. R. Schleyer, C. Maerker, A. Dransfeld, H. Jiao, and N. J. R. van Eikema Hommes, "Nucleus-independent chemical shifts: a simple and efficient aromaticity probe," Journal of the American Chemical Society, vol. 118, no. 26, pp. 6317-6318, 1996.

[36] W. Shen, M. Li, R. He, J. Zhang, and W. Lei, "The electronic and structural properties of nonclassical bicyclic thiophene: monomer, oligomer and polymer," Polymer, vol. 48, no. 13, pp. 3912-3918, 2007.

[37] J. E. Carpenter and F. Weinhold, "Analysis of the geometry of the hydroxymethyl radical by the "different hybrids for different spins" natural bond orbital procedure," Journal of Molecular Structure: THEOCHEM, vol. 169, pp. 41-62, 1988.

[38] A. E. Reed, L. A. Curtiss, and F. Weinhold, "Intermolecular interactions from a natural bond orbital, donor-acceptor viewpoint," Chemical Reviews, vol. 88, no. 6, pp. 899-926, 1988.

[39] J. P. Foster and F. Weinhold, "Natural hybrid orbitals," Journal of the American Chemical Society, vol. 102, no. 24, pp. 7211$7218,1980$.

[40] A. E. Reed, R. B. Weinstock, and F. Weinhold, "Natural population analysis," The Journal of Chemical Physics, vol. 83, no. 2, pp. 735-746, 1985.

[41] S. I. Gorelsky and A. B. P. Lever, "Electronic structure and spectra of ruthenium diimine complexes by density functional theory and INDO/S. Comparison of the two methods," Journal of Organometallic Chemistry, vol. 635, no. 1-2, pp. 187-196, 2001.

[42] Y. Fu, W. Shen, and M. Li, "Theoretical analysis on the electronic structures and properties of PPV fused with. Electron-withdrawing unit: monomer, oligomer and polymer," Polymer, vol. 49, no. 10, pp. 2614-2620, 2008.

[43] P. von Ragué Schleyer, H. Jiao, N. J. R. van Eikema Hommes, V. G. Malkin, and O. L. Malkina, "An evaluation of the aromaticity of inorganic rings: refined evidence from magnetic properties," Journal of the American Chemical Society, vol. 119, no. 51, pp. 12669-12670, 1997.

[44] P. Rague von Schleyer and H. Jiao, "What is aromaticity?," Pure and Applied Chemistry, vol. 68, no. 2, pp. 209-218, 1996.

[45] R. Kroon, M. Lenes, J. C. Hummelen, P. W. M. Blom, and B. de Boer, "Small bandgap polymers for organic solar cells (polymer material development in the last 5 years)," Polymer Reviews, vol. 48, no. 3, pp. 531-582, 2008.

[46] L. J. A. Koster, V. D. Mihailetchi, R. Ramaker, and P. W. M. Blom, "Light intensity dependence of open-circuit voltage of polymer:fullerene solar cells," Applied Physics Letters, vol. 86, no. 12, pp. 123509/1-123509/3, 2005.

[47] V. D. Mihailetchi, P. W. M. Blom, J. C. Hummelen, and M. T. Rispens, "Cathode dependence of the open-circuit voltage of polymer: fullerene bulk heterojunction solar cells," Journal of Applied Physics, vol. 94, no. 10, pp. 6849-6854, 2003.

[48] C. W. Bauschlicher Jr. and J. W. Lawson, "Current-voltage curves for molecular junctions: the effect of substituents," Physical Review B, vol. 75, article 115406, 2007.

[49] M. F. Lo, T. W. Ng, T. Z. Liu et al., "Limits of open circuit voltage in organic photovoltaic devices," Applied Physics Letters, vol. 96, no. 11, p. 113303, 2010.

[50] J. L. Bredas, D. Beljonne, V. Coropceanu, and J. Cornil, “Chargetransfer and energy-transfer processes in $\pi$-conjugated oligomers and polymers: a molecular picture," Chemical Reviews, vol. 104, no. 11, pp. 4971-5004, 2004.

[51] R. C. Haddon, T. Siegrist, R. M. Fleming, P. M. Bridenbaugh, and R. A. Laudise, "Band structures of organic thin-film transistor materials," Journal of Materials Chemistry, vol. 5, no. 10, pp. 1719-1724, 1995.

[52] J. Cornil, J. P. Calbert, and J. L. Brédas, "Electronic structure of the pentacene single crystal: relation to transport properties," Journal of the American Chemical Society, vol. 123, no. 6, pp. 1250-1251, 2001.

[53] Y. C. Cheng, R. J. Silbey, D. A. da Silva Filho, J. P. Calbert, J. Cornil, and J. L. Brédas, "Three-dimensional band structure and bandlike mobility in oligoacene single crystals: a theoretical investigation," The Journal of Chemical Physics, vol. 118, no. 8, pp. 3764-3774, 2003.

[54] J. G. Zhu, W. X. Zheng, J. G. Zheng, X. S. Sun, and H. T. Wang, Solid Physics, Science Press, Beijing, China, 2005.

[55] R. A. Marcus, "Electron transfer reactions in chemistry: theory and experiment (Nobel lecture)," Angewandte Chemie International Edition, vol. 32, no. 8, pp. 1111-1121, 1993.

[56] A. A. Voityuk, "Estimation of electronic coupling in $\pi$-stacked donor-bridge-acceptor systems: correction of the two-state model," The Journal of Chemical Physics, vol. 124, no. 6, article 064505, 2006.

[57] G. J. Kavarnos and N. J. Turro, "Photosensitization by reversible electron transfer: theories, experimental evidence, and examples," Chemical Reviews, vol. 86, no. 2, pp. 401-449, 1986. 


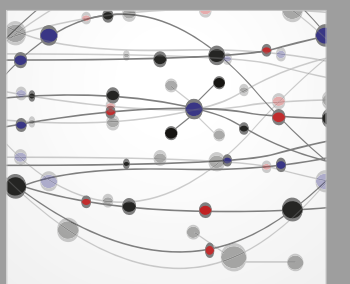

The Scientific World Journal
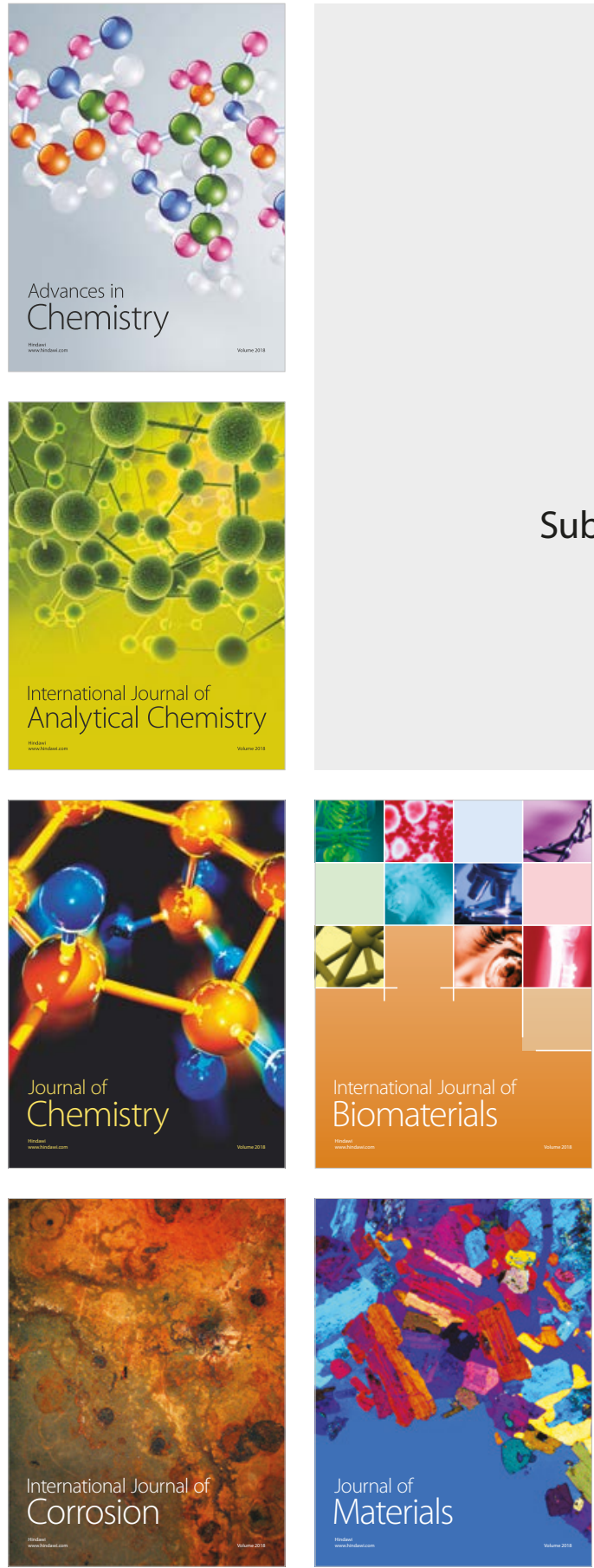

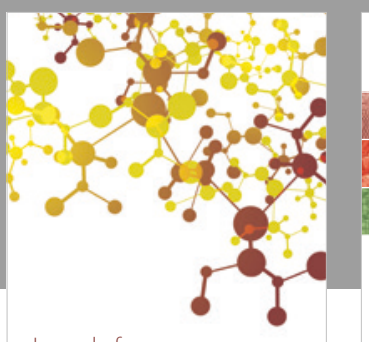

Journal of

Applied Chemistry
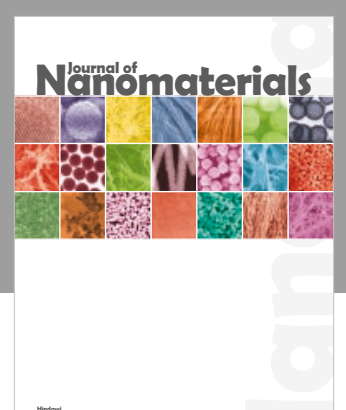

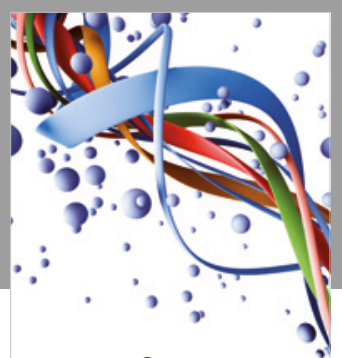

Scientifica

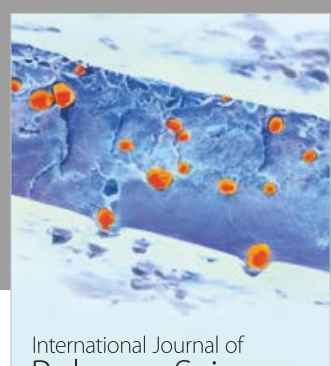

Polymer Science

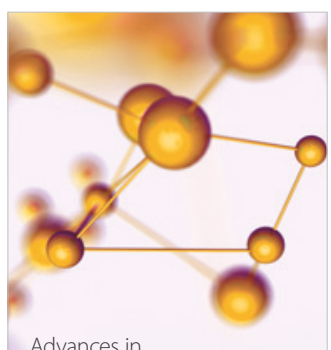

Physical Chemistry
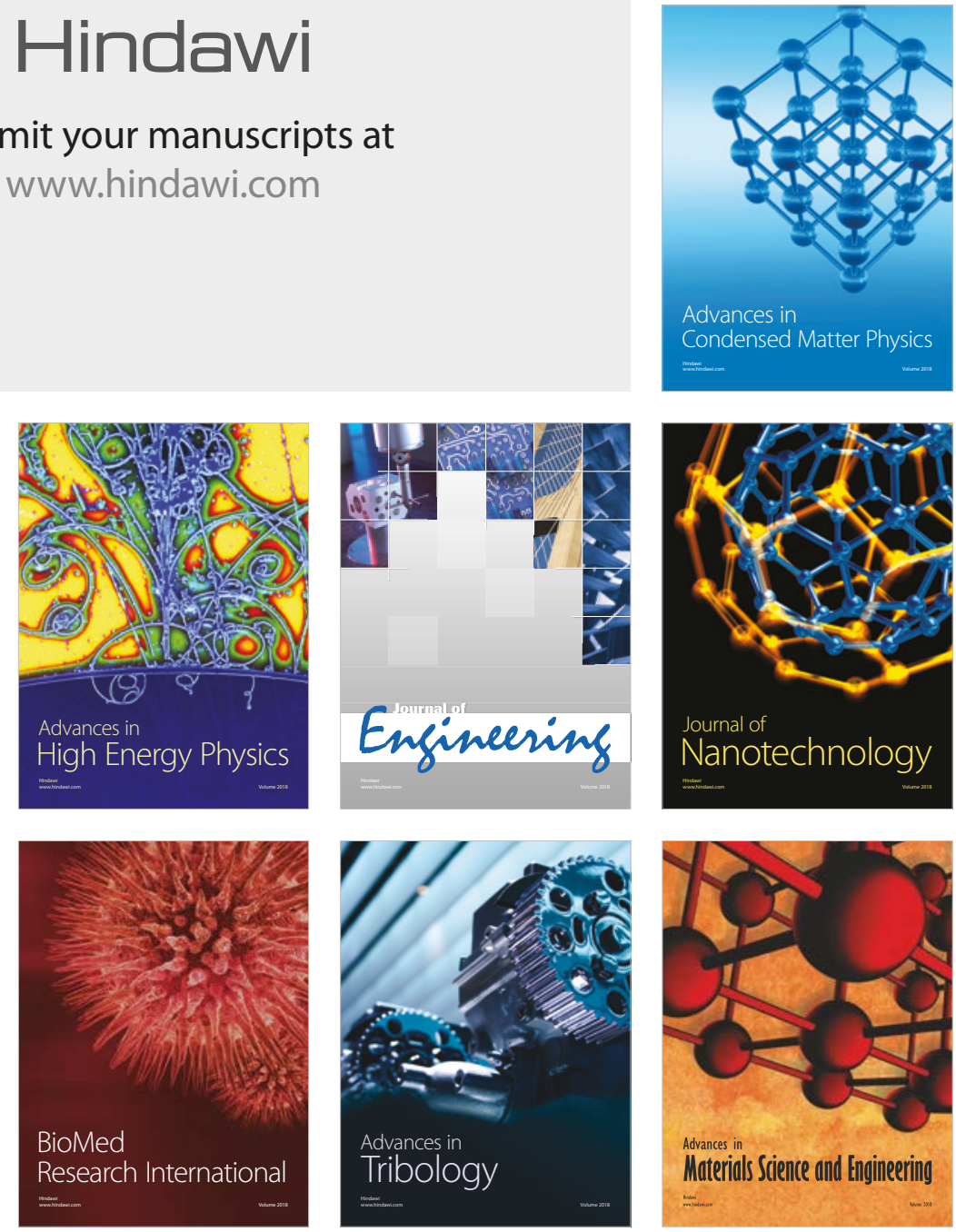